\title{
Selective elimination of the contact site A protein of Dictyostelium discoideum by gene disruption
}

\author{
Christiane Harloff, Günther Gerisch, and Angelika A. Noegel \\ Max-Planck-Institut für Biochemie, D-8033 Martinsried bei München, Federal Republic of Germany
}

The contact site A glycoprotein is a developmentally regulated cell-surface component expressed during the aggregation stage of Dictyostelium discoideum. This protein has been implicated in the EDTA-stable $\left(\mathrm{Ca}^{2+}\right.$ independent) type of cell adhesion of aggregating cells. The gene coding for the contact site $A$ protein was disrupted by homologous recombination, using a transformation vector that contained a 1.0-kb cDNA fragment as an insert. Transformants that did not express the protein were identified by colony immunoblotting. These transformants produced three truncated contact site A transcripts. One of them was controlled by the original contact site A promoter, as indicated by its strict developmental regulation and cAMP inducibility; the other two transcripts were transcribed from the actin 6 promoter of the vector. When cell adhesion was assayed in the transformants by agitating suspended cells in an agglutinometer, EDTA-stable adhesion was drastically reduced as compared to wild type, confirming that the contact site A glycoprotein acts as a cell-adhesion molecule. However, aggregation of the transformed cells on an agar surface was not remarkably altered. These results suggest that the contact site $\mathbf{A}$ glycoprotein is responsible for a 'fast' type of cell adhesion that is essential when aggregating cells are subjected to shear. When cells are not mechanically disturbed, a 'slow' type of adhesion mediated by other molecules is sufficient for their aggregation.

[Key Words: Homologous recombination; $\mathrm{Ca}^{2+}$-independent cell adhesion; Dictyostelium]

Received May 26, 1989; revised version accepted September 8, 1989.

Two types of cell adhesion can be distinguished in aggregating cells of Dictyostelium discoideum: EDTA-stable and EDTA-sensitive (Beug et al. 1973). The EDTAstable type of adhesion is developmentally regulated; it becomes detectable when the cells start to aggregate. At the same time, the contact site A (csA) glycoprotein becomes expressed (Müller and Gerisch 1978). The acquisition of EDTA-stable cell adhesiveness and csA expression are both stimulated by pulses of cAMP (Gerisch et al. 1985). The csA glycoprotein has been identified as a target site of antigen-binding fragments (Fab) of polyclonal antibodies that specifically block the EDTAstable type of cell adhesion (Gerisch et al. 1980). The csA molecule is modified by two types of oligosaccharide residues (Hohmann et al. 1987a), is highly sulfated at the $\mathrm{N}$-linked type 1 carbohydrate (Hohmann et al. 1985), and is distinguished from most of the other membrane proteins that are expressed between growth phase and cell aggregation by the presence of a phospholipid anchor (Stadler et al. 1989).

A nitrosoguanidine-induced mutant that lacks the csA protein has provided support for a function of this protein in EDTA-stable cell adhesion (Noegel et al. 1985). Additional evidence has been obtained from employing the purified glycoprotein or peptides representing portions of its sequence in adhesion studies (Kamboj et al.
1988). From these studies, it has been concluded that the protein portion of the csA directly mediates EDTAstable cell-to-cell adhesion. The csA protein is encoded by a single-copy gene (Noegel et al. 1986). In this paper we describe transformants in which the csA protein is eliminated by disruption of this gene and report that EDTA-stable cell adhesiveness is reduced substantially in these transformants. Selective inactivation of a gene by homologous recombination has been employed previously in $D$. discoideum for studies on two cytoskeletal proteins, myosin (DeLozanne and Spudich 1987) and $\alpha$ actinin (Witke et al. 1987). The finding that three transcripts are produced in the csA transformants, one controlled by the resident csA promoter and two by the actin promoter of the transformation vector, indicates that the vector has integrated more than once in the same gene.

\section{Results}

Design of a vector for homologous recombination with the csA gene

A transformation vector, $\mathrm{pDcsAl}$, was constructed by inserting a $1.0-\mathrm{kb}$ fragment of the cDNA clone c512 into the Dictyostelium vector pDNeoII (Witke et al. 1987). The fragment used as an insert contains the coding re- 


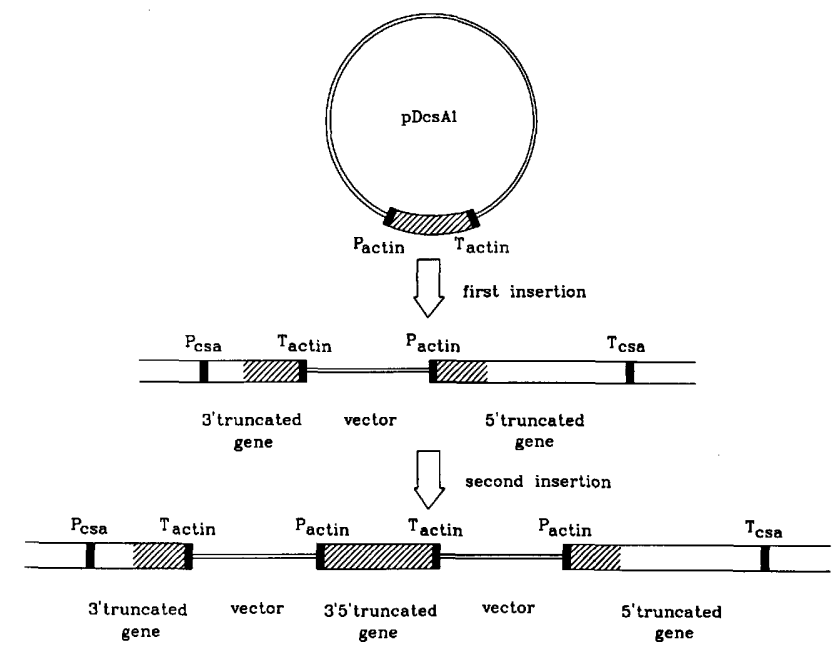

Figure 1. Diagram showing the truncated gene fragments that occur after insertion of one or two pDcsAl copies into the csA gene. Transcription of these fragments is driven either by the csa promoter $\left(\mathrm{P}_{\text {csa }}\right)$ or actin 6 promoter $\left(\mathrm{P}_{\text {actin }}\right)$, and is terminated by the actin 8 terminator $\left(T_{\text {actin }}\right)$ or csa terminator $\left(T_{\text {csa }}\right)$. The precise recombination site between vector sequences (hatched) and genomic sequences (open) may vary from one transformant to the other.

gion for the amino-terminal part of the csA protein up to valine in position 278 . Upstream of this region the fragment comprises 39 nucleotides of the $5^{\prime}$-noncoding region of the csA gene and $\sim 80$ additional nucleotides that are present in the 512 clone (Noegel et al. 1986) but are not found in the genomic sequence (C. Harloff, unpubl.). This additional sequence, which seems to be attached to the csA sequences as an artifact of the cDNA cloning procedure, contains four in-frame stop codons. Transcription of the insert is driven in the $\mathrm{pDcsAl}$ vector by the actin 6 promoter of $D$. discoideum, which is connected with a $5^{\prime}$ sequence of the actin 6 gene that includes the coding region for the first 8 amino-terminal amino acids. Transcription is terminated at the $3^{\prime}$ side of the insert by two tandemly repeated actin 8 terminators (Witke et al. 1987). For the selection of transformants, pDcsA1 contains the phosphotransferase gene conferring neomycin resistance, which is driven by the actin 15 promoter of $D$. discoideum. Because the vector does not contain an origin of replication, it will be multiplied only after integration into the genome.

Homologous recombination of the insert of the pDcsA1 vector with the resident csA gene will result in two fragments if the vector integrates only once. A third fragment derived from the insert of the vector will be produced if the vector integrates twice or more often. These three fragments will be separated from each other by vector sequences (Fig. 1). In the $5^{\prime}$ fragment the connection of the coding region with the csA promoter will be unimpaired. However, if the transcript encoded by this gene fragment is translated, a polypeptide missing 217 amino acids from the carboxy-terminal portion of the csA protein will be produced. The same polypeptides would be produced from the $1.0-\mathrm{kb}$ insert sequence of the vector if it were translated. Because the phospholipid anchor of the csA glycoprotein is attached to the carboxy-terminal region of the molecule /Stadler et al. 1989|, the polypeptide lacking this region could not be anchored to the membrane. The $3^{\prime}$ fragment of the gene could give rise to a transcript that contains the entire coding region, but this region is separated by four stop signals at the 5 ' end of the inserted cDNA from the actin 6 promoter and from the coding sequence for the aminoterminal amino acids of actin. Therefore, a restart of translation at the ATG start codon of the cDNA insert would be necessary.

\section{Isolation of transformants with a disrupted $\operatorname{cs} A$ gene}

Cells of $D$. discoideum strain $\mathrm{AX} 2$ were transformed with pDcsA1, and transformants resistant to G418 were cloned and screened by colony blotting with $\mathrm{mAb} 71$ (Fig. 2A,B). This antibody recognizes the protein moiety of the csA glycoprotein. Of a total of 600 transformants tested in two independent experiments, 19 colonies that did not react with the antibody were found. In a control transformation with the pDNeoII vector lacking the insert, all of the 300 transformants tested produced the csA protein (Fig. 2A).

From three transformants of one experiment and one transformant of the other, nuclear DNA was prepared and employed for Southern blotting. The DNA was cut with HindIII and EcoRI, and the blots were hybridized with nick-translated cDNA and genomic fragments (Fig. 3) to probe for csA gene sequences, and with linearized pUC19 DNA to probe for vector sequences. According to

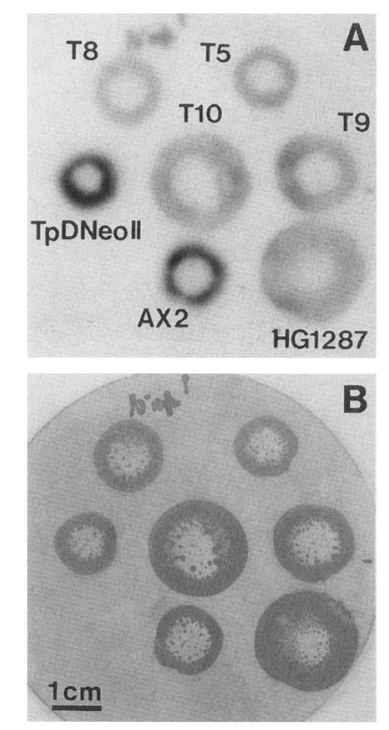

Figure 2. (A) Colony blot analysis of four transformants. The colony blot was labeled with ${ }^{125}$ I-labeled $\mathrm{mAb} 71$. Only aggregates of wild-type AX2 and the control transformant TpDNeoII were strongly labeled. $(B)$ Proteins of the same blot stained with Ponceau S. HG1287 is a derivative of the csA mutant HG693 induced by chemical mutagenesis (Noegel et al. 1985). It is used as a control to monitor background labeling in a csA-negative strain. 


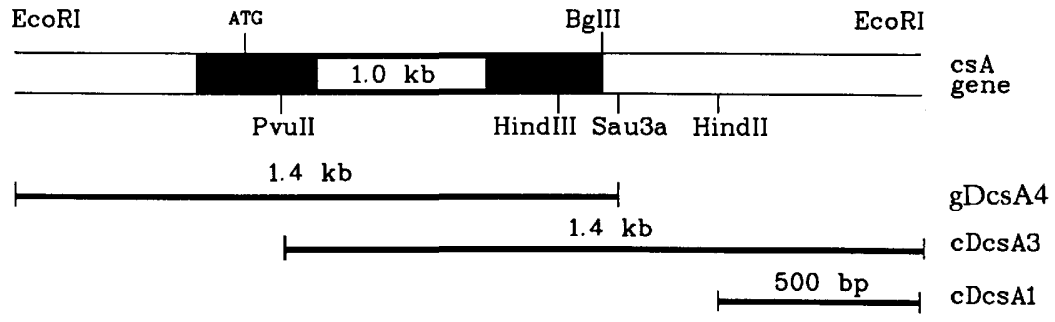

Figure 3. Location of $\operatorname{csA-DNA}$ probes used for Southern and Northern hybridizations. The insert of the transformation vector pDcsAl is shown as a solid box. cDcsAl and cDcsA3 represent fragments of the cDNA clone c512; gDcsA4 a genomic clone including $\sim 400$ bp of the $5^{\prime}$ untranslated region. The respective sizes of the probes are indicated. the diagram of Figure 4, the EcoRI digest can be used to demonstrate gene disruption by homologous recombination, and the HindIII digest can be used to exclude gross rearrangements of the csA gene or vector sequences and to make random integration of the vector into other regions of the genome unlikely.

Although the transcribed region of the csA gene contains no EcoRI site, the pDcsAl vector contains one site into which the $5^{\prime}$ end of the insert was cloned. Thus, if the vector integrates once into the gene, two EcoRI fragments will be expected to hybridize with full-length cDNA probes, and three different fragments will be expected to hybridize if the vector integrates at least twice. In EcoRI digests from wild-type AX2, a single fragment of $6.8 \mathrm{~kb}$ was recognized by csA cDNA probes of various lengths (Fig. 5, lanes 2, 9, and 11), and, as expected, no band was labeled with the pUC probe (Fig. 5, lanes 6 and 13). In the transformants, the $6.8 \mathrm{-kb}$ wild-type fragment was replaced by three other fragments of 5.8, 6.7, and 8.7 $\mathrm{kb}$ (Fig. 5, lanes 4 and 10), which correspond to those predicted from Figure 4, assuming that the csA gene was disrupted. The 6.7-kb EcoRI fragment was not recog- nized by the cDcsAl probe but was recognized by the pUC probe, which distinguishes the $6.7-\mathrm{kb}$ fragment of the transformants from the 6.8 -kb fragment of the wild type (Fig. 5, lanes 12 and 14). It is the presence of this $6.7-\mathrm{kb}$ fragment comigrating with the linearized transformation vector that shows that the vector integrated at least twice in the transformants.

\section{Three differently regulated transcripts are produced in the transformants}

The 1.9-kb message of the csA gene is strictly developmentally regulated in the untransformed $\mathrm{AX} 2$ strain. No transcript has been detected in growth-phase cells, and substantial expression has been found after $5-6 \mathrm{hr}$ of development in suspension cultures, shortly before the cells acquire full aggregation competence (Noegel et al. 1985). Figure 6 shows that no csA transcript corresponding to the $1.9-\mathrm{kb}$ mRNA of the wild type was produced in the transformants. Instead, three transcripts of $1.2,1.3$, and $2.2 \mathrm{~kb}$ were labeled using the insert of the transformation vector as a probe. The $1.3-$ and $2.2-\mathrm{kb}$

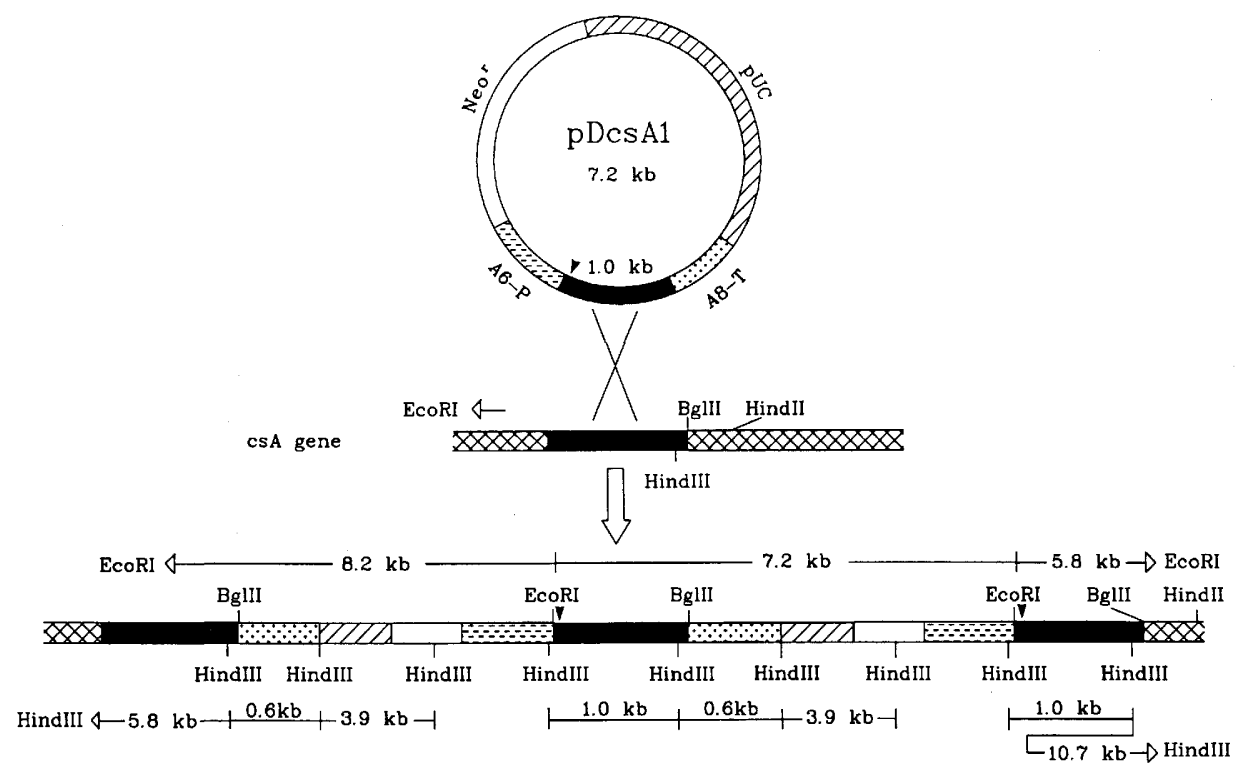

Figure 4. Diagram of restriction fragments expected after multiple integrations of the vector into the csA gene. The 1.0-kb fragment of clone c512 inserted into the EcoRI and BgIII sites of the pDNeoII vector and the corresponding region of the csA gene are indicated by solid boxes. The arrowheads point to the $3^{\prime}$ end of a 74-bp sequence in clone c512 that contains stop codons and is not represented in the csA gene. Sequences of the resident gene not present in the insert are crosshatched. Expected sizes of vector and genomic sequences are taken from published data (Noegel et al. 1986; Witke et al. 1987). (Bottom) Numbers above lines indicate the length of fragments drawn proportionately; numbers within lines indicate the length of fragments that are arbitrarily shortened. 


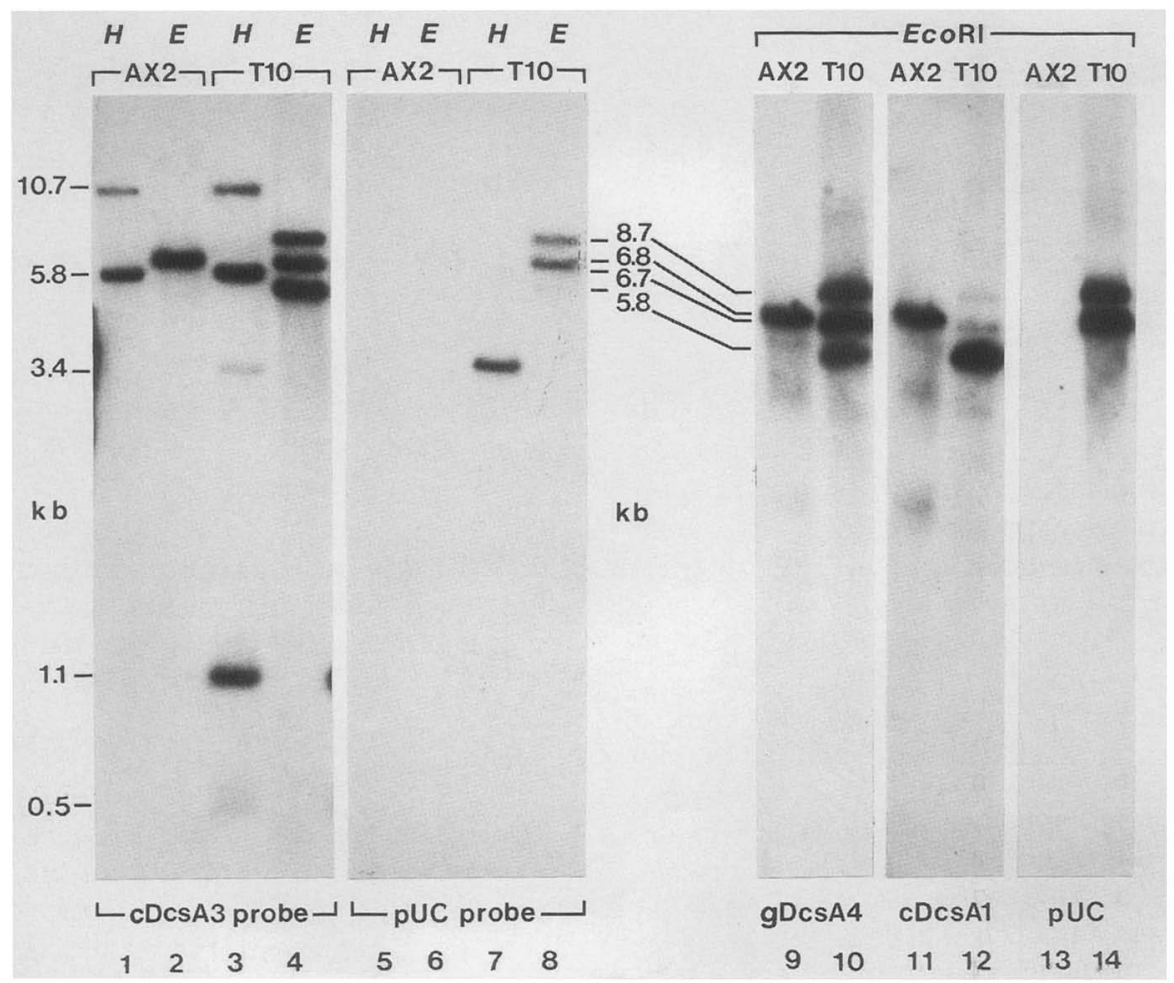

Figure 5. Southern blot analysis of nuclear DNA from wild-type AX2 and transformant T10. EcoRI (E)- and HindIII (H)digested DNAs were hybridized to the nick-translated DNA probe cDcsA3, gDcsA4, and cDcsAl (Fig. 3) and to linearized pUC19 sequences. Blots similar to those of lanes 1-8 were also done for the transformants T5, T8, and T9, and blots similar to those of lanes 9-14 were done for transformant T8. These transformants isolated independently of T10 gave the same results. All three EcoRI fragments were recognized by the cDcsA3 and gDcsA4 probes, which overlap with the insert of the vector. The cDcsAl probe, which represents 3 ' sequences of the csA gene not present in the insert (Fig. 3), recognized only the 5.8-kb fragment (lane 12), which was not labeled by the pUC probe (lanes 8 and 14). These results show that the $5.8-\mathrm{kb}$ fragment represents the $3^{\prime}$ portion of the disrupted gene. The 6.7- and $8.7-\mathrm{kb}$ fragments were not recognized by the cDcsAl probe but were recognized by the pUC probe (lanes $12,8,14)$. The $6.7-\mathrm{kb}$ fragment comigrated with the linearized transformation vector, which migrated somewhat faster than predicted from its calculated size of $7.2-\mathrm{kb}$ (Fig. 4). This indicates that the $6.7-\mathrm{kb}$ fragment contains the complete vector sequence. The size of the $8.7-\mathrm{kb}$ fragment is in good agreement with $8.2-\mathrm{kb}$, as predicted for the $5^{\prime}$ fragment of the disrupted gene (Fig. 4). HindIII cuts the resident csA gene at one site and the vector at four sites (Fig. 4). The 5.8- and 10.7-kb HindIII fragments of the wild-type AX2 gene were also found in the transformants (lanes 1 and 3). In addition, a $1.1-\mathrm{kb}$ and a faintly labeled $0.5-\mathrm{kb}$ fragment were recognized in the transformants by the cDcsA3 probe (lane 3). These fragments correspond to the 1.0-and 0.6-kb fragments derived from the vector. The $0.6-\mathrm{kb}$ fragment overlaps with the probe only by $\sim 50$ nucleotides, which explains its faint labeling. The pUC probe recognized a 3.4-kb band labeled in the transformants (lane 7), which corresponds to the 3.9 -kb vector fragment as predicted from Figure 4 . The fourth HindIII fragment, comprising part of the $\mathrm{Neo}^{\mathrm{R}}$ gene and the actin 6 promoter, is not recognized by either of the probes. The weak labeling of the 3.4-kb band in lane 3 is explained by contamination of the cDcsA3 probe with traces of pUC sequences.

transcripts were found in growth-phase cells, as well as in cells harvested after $6 \mathrm{hr}$ of development. In contrast, expression of the 1.2 -kb transcript depended as strictly on development as did expression of the 1.9-kb mRNA in the wild type. By the use of cDcsAl as a probe that does not hybridize to the insert but does bind to sequences at its $3^{\prime}$ side, only the $2.2-\mathrm{kb}$ transcript of the transformants was labeled (Fig. 6), showing that this large RNA is transcribed from the ${ }^{\prime}$ ' fragment of the gene. Accordingly, the 1.2- and 1.3-kb transcripts are the products of the two $3^{\prime}$-truncated fragments of the csA gene, as indicated in Figure 1.

The actin 6 gene is known to be expressed in growthphase cells. During development, the mRNA levels have been found to increase only transiently during the first 2-5 hr (Romans et al. 1985; Knecht et al. 1986). In ac- cord with the activity of the actin 6 promoter during growth and development, the $2.2-\mathrm{kb}$ transcript of the $3^{\prime}$ fragment of the gene that is driven by this promoter is expressed in growth-phase, as well as in developing, cells (Fig. 6). The same is true for the $1.3-\mathrm{kb}$ transcript, which therefore appears to represent the product of the $3^{\prime}$-, 5'-truncated gene (Fig. 1). Consequently, the strictly regulated $1.2-\mathrm{kb}$ transcript is thought to be transcribed from the 3 -truncated gene driven by the endogenous csA promoter. To confirm this hypothesis, regulation of the transcripts by pulses of cAMP was tested. Expression of the csA gene strongly depends on pulsatile cAMP signals (Noegel et al. 1985), whereas no regulation by cAMP is known for the actin 6 gene. The AX2 strain, from which the transformants are derived, normally produces its own cAMP signals but becomes dependent 


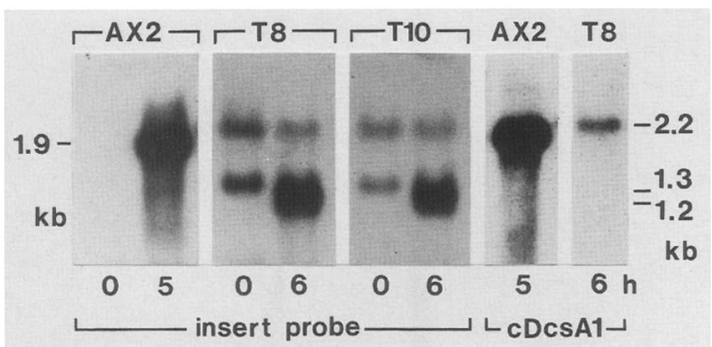

Figure 6. Northern blot analysis of total RNA from wild-type AX2 and transformants T8 and T10. Cells were harvested either during exponential growth in nutrient medium at a density of $3 \times 10^{6}$ cells $/ \mathrm{ml}(0 \mathrm{hr})$ or after $5-6 \mathrm{hr}$ of starvation in phosphate buffer. The amount of RNA per lane was $10 \mu \mathrm{g}$ for AX2 and $20 \mu \mathrm{g}$ for T8 and T10. Nick-translated insert of the pDcsA1 vector and cDcsAl were used as probes.

on exogenous cAMP signals to fully acquire aggregation competence if the cells are grown up to the late stationary phase before starvation in a nutrient-free buffer (Gerisch et al. 1975). Therefore, cells of transformant T10 were harvested at the late stationary phase and then either pulsed or not treated with cAMP, to identify cAMP-controlled transcripts. Figure 7 shows that the 1.3- and 2.2-kb transcripts were expressed independently of whether or not these cells were stimulated by cAMP, whereas expression of the $1.2-\mathrm{kb}$ transcript was strongly enhanced by the cAMP pulses.

\section{Lack of the csA protein strongly affects EDTA-stable cell adhesiveness}

To confirm that the transformants were deficient in csA production, total cellular proteins of 10 of the transformants were separated by SDS-PAGE and blotted for labeling with mAb 294, which recognizes an epitope on the csA polypeptide other than the one recognized by $\mathrm{mAb} 71$, the antibody used for selection of the transformants. No labeled band was observed with mAb 294 in either of the transformants tested, as shown for transfor-

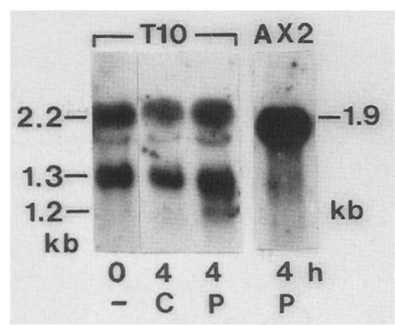

Figure 7. Northern blot analysis of total RNA from AX2 and transformant cells grown up to the late stationary phase and subsequently pulsed or unpulsed with cAMP. Cells were harvested from nutrient medium at a density of $1.2 \times 10^{7}$ cells $/ \mathrm{ml}$ and either used directly for RNA isolation $(0 \mathrm{hr})$ or after $4 \mathrm{hr}$ of starvation in phosphate buffer, with $(\mathrm{P})$ or without $(\mathrm{C})$ stimulation of development by pulses of cAMP. (AX2 and T10) $10 \mu \mathrm{g}$ and $40 \mu \mathrm{g}$, respectively, of RNA were loaded per lane. Nicktranslated insert of the transformation vector $\mathrm{pDcsAl}$ was used as a probe.

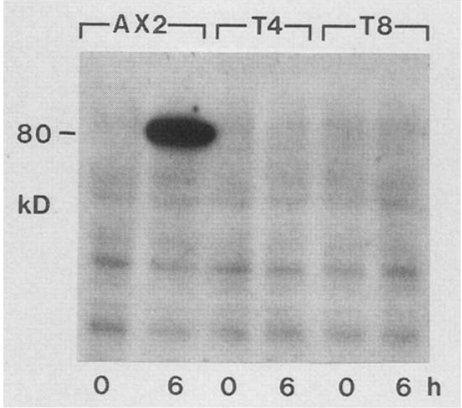

Figure 8. Immunoblot of proteins from wild-type AX2 and two transformants separated by SDS-PAGE. Cells were harvested either from nutrient medium $(0 \mathrm{hr})$ or after $6 \mathrm{hr}$ of starvation in phosphate buffer. Total proteins of $10^{6}$ cells were applied per lane, and the csA protein was labeled with ${ }^{125}$ I-labeled $\mathrm{mAb} 294$. The blots were overexposed until background labeling was seen to demonstrate undetectability of $\operatorname{csA}$ in the transformants.

mants T4 and T8 in Figure 8. Identical blots were also labeled with mAb 210, which reacts with an epitope of type 2 carbohydrate residues that are present on csA, as well as on other $D$. discoideum glycoproteins (Hohmann et al. 1987a). These carbohydrate residues are required for protecting the csA protein against degradation by proteases (Hohmann et al. 1987b). Antibody mAb 210 visualized glycoproteins other than csA in the transformants, showing that absence of the csA protein did not result from a defect in glycosylation. However, the $80-\mathrm{kD}$ band of the csA glycoprotein was missing /data not shown). The absence of any recognizable csA protein in the transformed cells indicates that the transcripts encoded by the $3^{\prime}$ portion of the gene are not translatable, although they contain the entire coding region. Thus, apparently no restart occurs behind the stop codons implemented into the insert.

For further investigations, the transformants T8 and $\mathrm{T} 10$, one from each of the two independent experiments, were chosen. To test whether a protein is synthesized that lacks the carboxy-terminal region and therefore cannot be anchored to the cell membrane, culture supernatants from T8 and T10 were tested for incomplete cs A molecules that are released into the medium. No labeled csA fragment was identified by mAb 294 in the extracellular fluid that was collected from developing cells at intervals during the first $24 \mathrm{hr}$ of starvation /data not shown). Thus, there is no evidence for a stable protein encoded by the $5^{\prime}$ fragment of the disrupted gene.

Targeted disruption of a gene is a straightforward way to identify the function of its product; however, coselection of spontaneous mutations is a serious source of errors in interpreting the transformation experiments. The presence of mutations that cause a general block in developmental gene expression was excluded by examining the product of the D-2 gene in the transformants. The D-2 gene is expressed during early development, and its expression is enhanced by pulsatile cAMP signals, similar to that of the csA gene (Mann et al. 1988 ). Figure 9 shows that the $57-\mathrm{kD}$ protein encoded by 


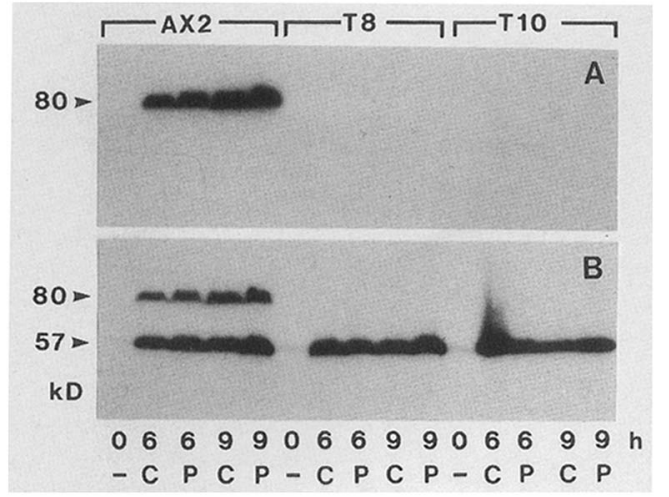

Figure 9. Lack of the $80-\mathrm{kD}$ csA glycoprotein in two transformants even after extended development and stimulation by cAMP pulses, and normal expression of the $57-\mathrm{kD}$ product of the D-2 gene. Exponentially growing cells were harvested from nutrient medium $(0 \mathrm{hr})$ and subsequently starved for 6 or $9 \mathrm{hr}$ in phosphate buffer, with $(\mathrm{P})$ or without $(\mathrm{C})$ pulsatile application of cAMP. Total cellular proteins of $10^{6}$ cells/lane were separated by SDS-PAGE, blotted, and labeled with ${ }^{125}$ I-labeled $\mathrm{mAb}$ 294 for csA $(A)$ and subsequently with ${ }^{125}$ I-labeled mAb 418 for the D-2 protein $(B)$. The blots show normal developmental regulation in the transformants by expression of the $D-2$ protein and absence of the csA protein under all conditions.

the D-2 gene was normally synthesized during early development in $\mathrm{T} 8$ and $\mathrm{T} 10$. The finding that development of both transformants in agar plate cultures was not obviously altered also argues against a general block in development. Like the parent strain $\mathrm{AX} 2$, transformants $\mathrm{T} 8$ and $\mathrm{T} 10$ formed streams of aggregating cells pointing toward aggregation centers and developed into fruiting bodies that were normally organized into stalks and spore heads (Fig. 10). Finally, the study of two independent transformants largely increased the probability that phenotypic changes common to both transformants are caused by the disruption of the csA gene.

Formation of EDTA-stable cell adhesion was examined in both $\mathrm{T} 8$ and $\mathrm{T} 10$ by subjecting cells to constant shear in an agglutinometer. In the presence of $10 \mathrm{~mm}$ EDTA, the parent AX2 strain formed large aggregates and the transformants formed very small ones, with many cells remaining single (Fig. 11). These results indicate that $\mathrm{Ca}^{2+}$-independent cell adhesion was strongly reduced in the transformants, although it was not completely abolished.

\section{Discussion}

The csA glycoprotein is absent from growth-phase cells and is maximally expressed on the surface of cells in the aggregation stage. The finding that the EDTA-stable adhesiveness of these cells is substantially reduced in transformants that lack the csA protein as a consequence of homologous recombination provides final proof that the csA glycoprotein is a cell-adhesion molecule. It also confirms previous results that were obtained with a csA-negative mutant produced by chemical mutagenesis, which showed that this glycoprotein is not
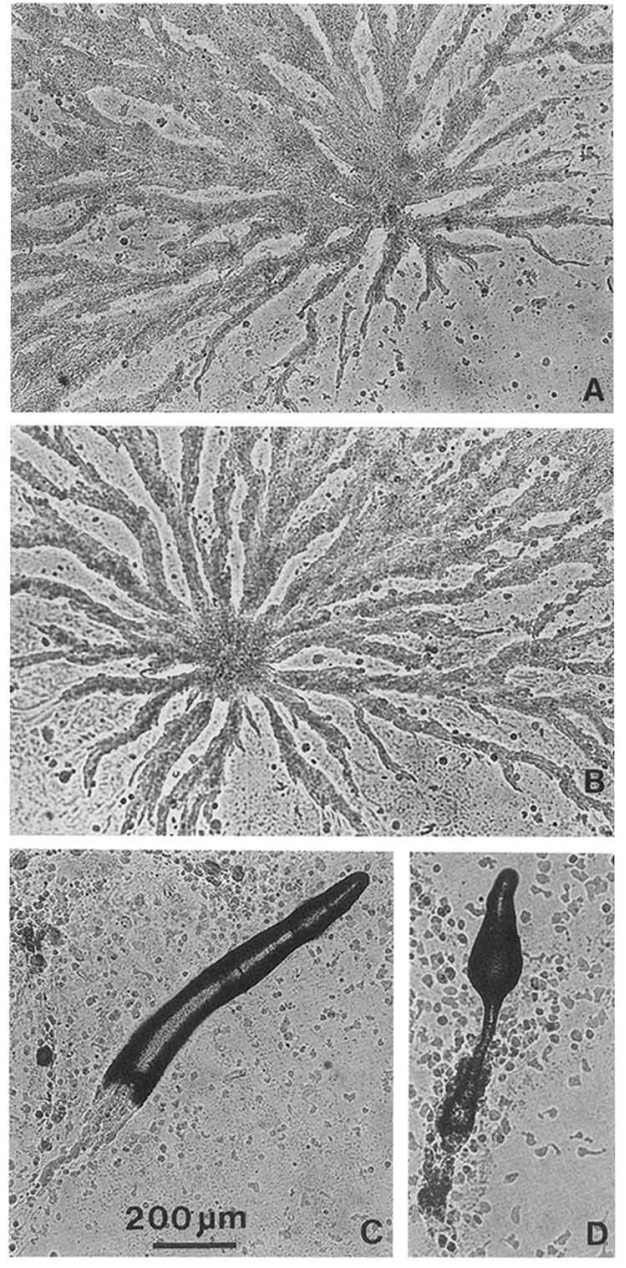

Figure 10. Control aggregate of the AX2 strain $(A)$, and aggregate $(B)$, slug $(C)$, and late culmination stage $(D)$ of transformant T10 on agar plate cultures.

the only cell-adhesion molecule acting during the aggregation stage (Noegel et al. 1985). Both this mutant and the transformants used in the present study are still capable of aggregating on an agar surface by forming streams of cohering cells.

It has been suggested that the csA glycoprotein is responsible for a fast type of cell adhesion and that its phospholipid anchor guarantees the high mobility in the plasma membrane that enables this protein to rapidly match another molecule at an adjacent cell surface (Stadler et al. 1989). The postulated fast type of cell adhesion is thought to be assayed when cells are subjected to shear, as, for example, in the agglutinometer that has been used for the determination of EDTA-stable cell adhesion, but not to be essential for undisturbed cell aggregation on the surface of an agar plate. Our results also show that even when cells are subjected to the shear forces applied in the agglutinometer, the EDTA-stable cell adhesion is not completely abolished. On the basis of these results, it is demanding to search for other cellsurface components that supplement the csA glycoprotein in its function as a cell-adhesion molecule of aggre- 


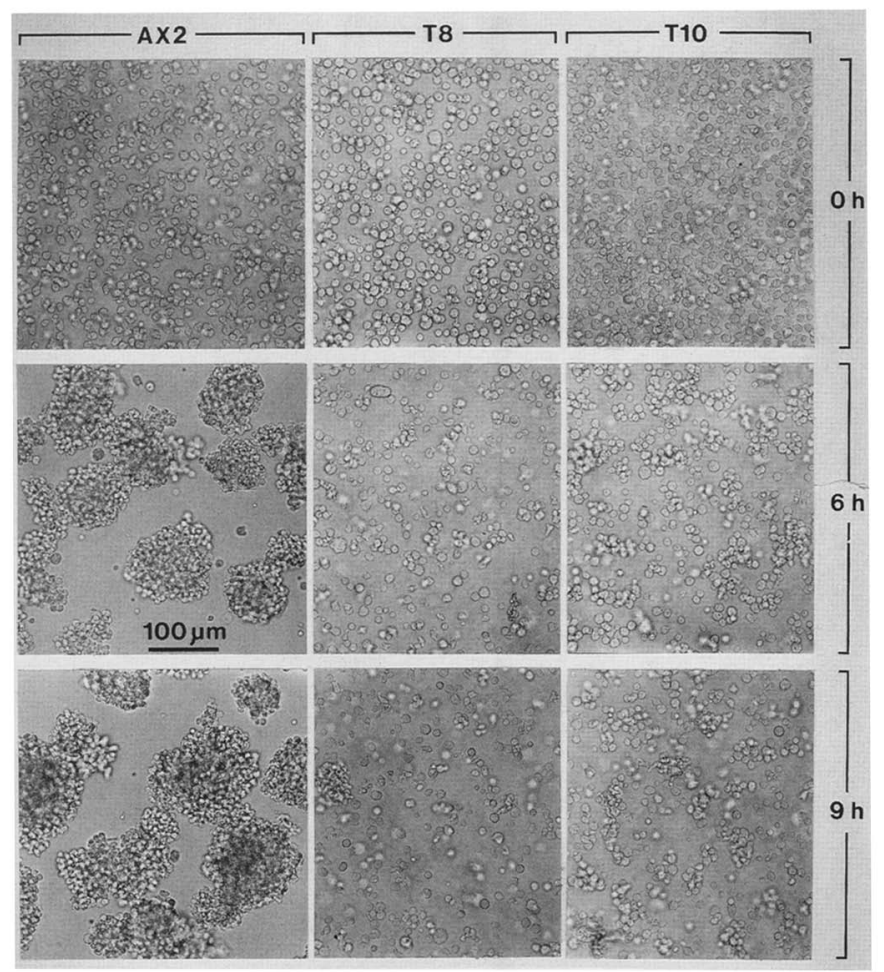

Figure 11. Agglutination of wild-type AX2 and transformant T8 and T10 cells in the presence of EDTA. Cells were harvested from nutrient medium at the exponential growth phase and used immediately $(0 \mathrm{hr})$ or starved for 6 or $9 \mathrm{hr}$ in phosphate buffer with stimulation by cAMP pulses. Washed cells were subjected to constant shear using an agglutinometer. Cells and agglutinates of AX2 (left), T8 (middle), and T10 (right) were photographed after agitation in the agglutinometer for $1 \mathrm{hr}$ in the presence of $10 \mathrm{~mm}$ EDTA.

gating cells. At present, there are two candidates: One is a 24-kD glycoprotein that is expressed early in development (Loomis 1988); the other is a $130-\mathrm{kD}$ glycoprotein that shares a phospholipid anchor with the csA glycoprotein (Stadler et al. 1989).

By multiple integration of the vector, three different fragments of the csA gene were produced in our experiments (Fig. 5). Transcription of two of them was driven by the actin 6 promoter of the vector, and one by the endogenous promoter of the gene. In Northern blots, transcripts from all three fragments could be detected. The transcript driven by the resident csA promoter was still under strict developmental control, and its expression was enhanced by cAMP signals as it is known for the normal csA transcript (Noegel et al. 1985). Because the gene fragment that encodes this transcript is truncated at its $3^{\prime}$ end, lacking part of the coding region and the entire 3 '-flanking sequences, these results imply that the specificity of transcript regulation is maintained in the separated $5^{\prime}$ portion of the gene. However, we never observed amounts of the $3^{\prime}$-truncated transcript in the transformants that were comparable to those of the complete csA transcript in the wild type. It must be concluded, therefore, that the $3^{\prime}$ half of the gene is required for full expression or stability of the transcript. With the data available, we cannot decide whether this is due to 3 '-enhancer sequences, to polyadenylation sites that are lacking in the $3^{\prime}$-truncated transcripts, or to other transcribed sequences that may increase the stability of the RNA.

The transformation vector used was constructed so that two of the transcripts encoded a fragment of the csA protein lacking 217 amino acids of the polypeptide sequence, including the carboxyl-terminus, which is required for anchoring the protein in the membrane. Thus, no functional cell-adhesion protein can be produced by these transcripts. In fact, no soluble csA protein was found within the cells or in the extracellular medium, suggesting that the nonpolyadenylated RNA is not translated or the incomplete polypeptide is degraded because of incorrect folding. The third csA transcript produced in the transformants includes the entire coding and 3 '-flanking regions, including the polyadenylation sites; however, this transcript contains several stop codons that separate the ATG start site of the csAcoding sequence from the few actin 6 codons linked to the promoter. The absence of any detectable csA protein in the transformants indicates that no restart occurs after the stop codons, in accord with results obtained in other eukaryotes (Kozak 1989).

The disruption of the csA gene reported in this paper is the third known example in which a Dictyostelium gene has been inactivated by homologous recombination with a fragment of that gene cloned into a transformation vector. In contrast to the previously reported examples of myosin II (DeLozanne and Spudich 1987) and $\alpha$-actinin (Witke et al. 1987), which concern constitutively expressed genes, this example shows inactivation of a strictly developmentally regulated gene that is not detectably expressed at the growth phase during which the recombination occurs. This result shows that targeted mutagenesis can be applied to studies on the function of genes in Dictyostelium that are expressed in a certain developmental stage or in a specific cell type.

\section{Materials and methods \\ Dictyostelium strains, culture conditions, and measurement of cell adhesion}

For transformation, the $D$. discoideum strain AX2-214 was used. $\mathrm{AX} 2$ and the transformants were cultivated at $23^{\circ} \mathrm{C}$, either on SM agar plates with Klebsiella aerogenes (Williams and Newell 1976), on nutrient agar plates with Escherichia coli $\mathrm{B} / 2$ (Noegel et al. 1985), or axenically in liquid nutrient medium containing 1.8\% maltose (Watts and Ashworth 1970). Exponentially growing cells were harvested from this medium at a density of $\sim 3 \times 10^{6}$ cells $/ \mathrm{ml}$; cells grown to the late stationary phase were harvested at a density of $\sim 1.2 \times 10^{7}$ cells $/ \mathrm{ml}$. The cells were washed and adjusted to $10^{7}$ cells $/ \mathrm{ml}$ in $17 \mathrm{mM}$ Soerensen phosphate buffer (pH 6.0), and shaking was continued on a gyratory shaker at $150 \mathrm{rpm}$. For stimulation of development by cAMP pulses, cAMP was applied every $6 \mathrm{~min}$ at a final concentration of $20 \mathrm{nM}$ during the starvation period. Cell adhesion was determined using an agglutinometer assay, as described by Beug et al. (1973) and Bozzaro et al. (1987). 
Vector construction and transformation

In the pDNeoII vector, transcription of the phosphotransferase gene that confers neomycin resistance to the transformants is driven by the actin 15 promoter of $D$. discoideum (Witke et al. 1987). A 1.0-kb EcoRI-BgIII fragment, representing the $5^{\prime}$ portion of csA clone c512 (Noegel et al. 1986), was cloned in sense orientation into the EcoRI and BglII sites of this vector. The resulting construct was named pDcsAl. Cells were transformed essentially according to Nellen et al. (1984); transformants were selected for G418 resistance using $20 \mu \mathrm{g} / \mathrm{ml}$ of Geneticin (Sigma) and cloned by spreading dilutions onto SM agar plates containing $K$. aerogenes. Cells from single colonies were picked and transferred onto new plates in an ordered array and allowed to grow until the colonies had reached a diameter of $\sim 7 \mathrm{~mm}$ (Wallraff et al. 1986). Transformants TpDcsA1-4 to TpDcsA1-9 were obtained from one transformation experiment, and TpDcsA1-10 from a second, independent one. These transformants are designated $\mathrm{T} 4, \mathrm{~T} 5, \mathrm{~T} 8, \mathrm{~T} 9$, and $\mathrm{T} 10$, respectively.

\section{Colony-blotting, gel electrophoresis, and immunoblotting}

Colonies derived from cloned cells were blotted onto BA $85 \mathrm{ni}$ trocellulose filters (Schleicher \& Schüll, 3354 Dassel, FRG). Cells were lysed by freezing and thawing and washed in a Tris/ Tween $/ \mathrm{NaCl}$ buffer according to Wallraff et al. (1986). The blots were then incubated for $\sim 2 \mathrm{hr}$ with ${ }^{125}$ I-labeled $\mathrm{mAb} 71$, followed by autoradiography. SDS-polyacrylamide electrophoresis was performed in $10 \%$ gels according to Laemmli (1970). Total protein of cells growing in liquid medium was prepared by boiling cells in sample buffer containing $1 \%$ SDS. Proteins in the supernatant of starving cells were precipitated by adding 9 volumes of acetone precooled to $-20^{\circ} \mathrm{C}$ (Stadler et al. 1989). The pellet was resuspended and boiled in sample buffer, and the equivalent of $5 \times 10^{6}$ cells was loaded on SDS gels. Proteins were blotted from the gel onto nitrocellulose and labeled with ${ }^{125}$ I-labeled mAb 294, ${ }^{125}$ I-labeled mAb 210, or ${ }^{125 I-l a b e l e d ~ m A b}$ 418.

\section{Isolation and hybridization of DNA and RNA}

DNA and RNA were prepared as described by Witke et al. (1986). Hybridizations with ${ }^{32} \mathrm{P}$-labeled nick-translated cDNA probes were done for $18-20 \mathrm{hr}$ at $37^{\circ} \mathrm{C}$ in $50 \%$ formamide and $2 \times$ SSC (Mehdy et al. 1983). The filters were first washed at room temperature with $2 \times \mathrm{SCC}$ and $0.01 \%$ SDS and, then, under stringent conditions for $1 \mathrm{hr}$ at $37^{\circ} \mathrm{C}$ in $50 \%$ formamide wash buffer.

\section{Acknowledgments}

We thank J. Stadler and B. Drexl for iodinated antibodies, E. Wallraff for strain HG1287, W. Röwekamp for the D-2 fusion protein against which mAb 418 was raised, and B. Book for organizing the manuscript.

\section{References}

Beug, H., F.E. Katz, and G. Gerisch. 1973. Dynamics of antigenic membrane sites relating to cell aggregation in Dictyostelium discoideum. J. Cell Biol. 56: 647-658.

Bozzaro, S., R. Merkl, and G. Gerisch. 1987. Cell adhesion: Its quantification, assay of the molecules involved, and selection of defective mutants in Dictyostelium and Polysphondylium. Methods Cell Biol. 28: 359-385.

DeLozanne, A. and J.A. Spudich. 1987. Disruption of the Dic- tyostelium myosin heavy chain gene by homologous recombination. Science 236: 1086-1091.

Gerisch, G., U. Weinhart, G. Bertholdt, M. Claviez, and J. Stadler. 1985. Incomplete contact site A glycoprotein in HL220, a modB mutant of Dictyostelium discoideum. I. Cell Sci. 73: 49-68.

Gerisch, G., H. Fromm, A. Huesgen, and U. Wick. 1975. Control of cell-contact sites by cyclic AMP pulses in differentiating Dictyostelium cells. Nature 255: 547-549.

Gerisch, G., H. Krelle, S. Bozzaro, E. Eitle, and R. Guggenheim. 1980. Analysis of cell adhesion in Dictyostelium and Polysphondylium by the use of Fab. In Cell adhesion and motility, (ed. A.S.G. Curtis and J.D. Pitts), pp. 293-307. (Third Symposium of the British Society for Cell Biology/ Cambridge University Press, Cambridge.

Hohmann, H.-P., G. Gerisch, R.W.H. Lee, and W.B. Huttner. 1985. Cell-free sulfation of the contact site A glycoprotein of Dictyostelium discoideum and of a partially glycosylated precursor. J. Biol. Chem. 260: 13869-13878.

Hohmann, H.-P., S. Bozzaro, M.Yoshida, R. Merkl, and G. Gerisch. 1987a. Two-step glycosylation of the contact site A protein of Dictyostelium discoideum and transport of an incompletely glycosylated form to the cell surface. $J$. Biol. Chem. 262: 16618-16624.

Hohmann, H.-P., S. Bozzaro, R. Merkl, E. Wallraff, M. Yoshida, U. Weinhart, and G. Gerisch. 1987b. Post-translational glycosylation of the contact site A protein of Dictyostelium discoideum is important for stability but not for its function in cell adhesion. $E M B O$ \%. 6: 3663-3671.

Kamboj, R.K., L.M. Wong, T.Y. Lam, and C.-H. Siu. 1988. Mapping of a cell-binding domain in the cell adhesion molecule gp80 of Dictyostelium discoideum. I. Cell Biol. 107: 18351843.

Knecht, D.A., S.M. Cohen, W.F. Loomis, and H.F. Lodish. 1986. Developmental regulation of Dictyostelium discoideum actin gene fusions carried on low-copy and high-copy transformation vectors. Mol. Cell. Biol. 6: 3973-3983.

Kozak, M. 1989. The scanning model for translation: An update. J. Cell Biol. 108: 229-24l.

Laemmli, U.K. 1970. Cleavage of structural proteins during the assembly of the head of bacteriophage T4. Nature 227: 680685.

Loomis, W.F. 1988. Cell-cell adhesion in Dictyostelium discoideum. Dev. Genet. 9: 549-559.

Mann, S.K.O., C. Pinko, and R.A. Firtel. 1988. Regulation of Dictyostelium early gene expression in cAMP bypass mutants. Dev. Biol. 130: 406-410.

Mehdy, M.C., D. Ratner, and R.A. Firtel. 1983. Induction and modulation of cell-type-specific gene expression in Dictyostelium. Cell 32: 763-771.

Müller, K. and G. Gerisch. 1978. A specific glycoprotein as the target site of adhesion blocking Fab in aggregating Dictyostelium cells. Nature 274: 445-449.

Nellen, W., C. Silan, and R.A. Firtel. 1984. DNA-mediated transformation in Dictyostelium discoideum: Regulated expression of an actin gene fusion. Mol. Cell. Biol. 4: 28902898.

Noegel, A., G. Gerisch, J. Stadler, and M. Westphal. 1986. Complete sequence and transcript regulation of a cell adhesion protein from aggregating Dictyostelium cells. EMBO I. 5: $1473-1476$.

Noegel, A., C. Harloff, P. Hirth, R. Merkl, M. Modersitzki, J. Stadler, U. Weinhart, M. Westphal, and G. Gerisch. 1985. Probing an adhesion mutant of Dictyostelium discoideum with cDNA clones and monoclonal antibodies indicates a specific defect in the contact site A glycoprotein. $E M B O I$. 4: 3805-3810. 
Romans, P., R.A. Firtel, and C.L. Saxe III. 1985. Gene-specific expression of the actin multigene family of Dictyostelium discoideum. J. Mol. Biol. 186: 337-355.

Stadler, J., T.W. Keenan, G. Bauer, and G. Gerisch. 1989. The contact site A glycoprotein of Dictyostelium discoideum carries a phospholipid anchor of a novel type. EMBO $\mathrm{J}$. 8: $371-377$.

Wallraff, E., M. Schleicher, M. Modersitzki, D. Rieger, G. Isenberg, and G. Gerisch. 1986. Selection of Dictyostelium mutants defective in cytoskeletal proteins: Use of an antibody that binds to the ends of $\alpha$-actinin rods. EMBO I. 5: 61-67.

Watts, D.J. and J.M. Ashworth. 1970. Growth of myxamoebae of the cellular slime mould Dictyostelium discoideum in axenic culture. Biochem. I. 119: 171-174.

Williams, K.L. and P.C. Newell. 1976. A genetic study of aggregation in the cellular slime mould Dictyostelium discoideum using complementation analysis. Genetics 82: 287307.

Witke, W., W. Nellen, and A. Noegel. 1987. Homologous recombination in the Dictyostelium $\alpha$-actinin gene leads to an altered mRNA and lack of the protein. EMBO I. 6: 41434148.

Witke, W., M. Schleicher, F. Lottspeich, and A. Noegel. 1986. Studies on the transcription, translation, and structure of $\alpha$ actinin in Dictyostelium discoideum. I. Cell Biol. 103: 969-975. 


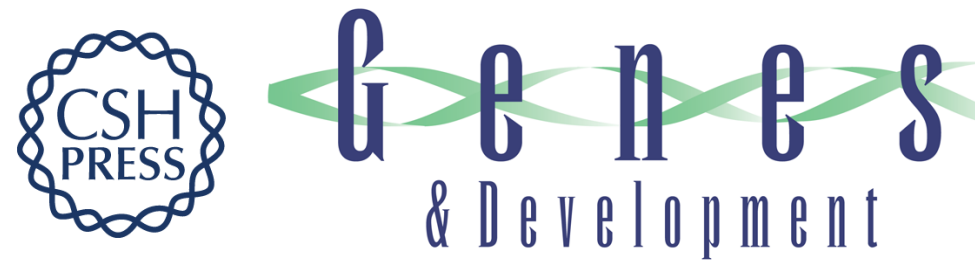

\section{Selective elimination of the contact site A protein of Dictyostelium discoideum by gene disruption.}

C Harloff, G Gerisch and A A Noegel

Genes Dev. 1989, 3:

Access the most recent version at doi:10.1101/gad.3.12a.2011

References This article cites 26 articles, 12 of which can be accessed free at:

http://genesdev.cshlp.org/content/3/12a/2011.full.html\#ref-list-1

License

Email Alerting

Service

Receive free email alerts when new articles cite this article - sign up in the box at the top right corner of the article or click here.

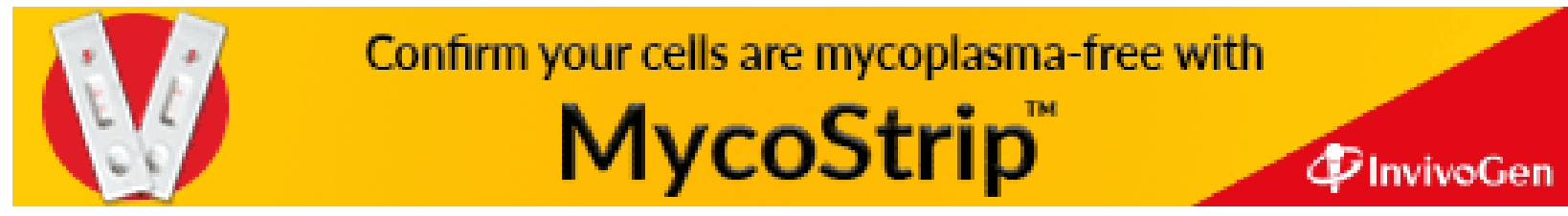

